\title{
Facilitating action planning within audit and feedback interventions: a mixed- methods process evaluation of an action implementation toolbox in intensive care
}

Wouter T. Gude ${ }^{1 *+} \mathbb{D}$, Marie-José Roos-Blom ${ }^{1,3+}$, Sabine N. van der Veer ${ }^{4}$, Dave A. Dongelmans ${ }^{2}$, Evert de Jonge ${ }^{5}$, Niels Peek ${ }^{4,6}$ and Nicolette F. de Keizer ${ }^{1,3}$

\begin{abstract}
Background: Audit and feedback (A\&F) is more effective if it facilitates action planning, but little is known about how best to do this. We developed an electronic A\&F intervention with an action implementation toolbox to improve pain management in intensive care units (ICUs); the toolbox contained suggested actions for improvement. A head-to-head randomised trial demonstrated that the toolbox moderately increased the intervention's effectiveness when compared with A\&F only.

Objective: To understand the mechanisms through which A\&F with action implementation toolbox facilitates action planning by ICUs to increase A\&F effectiveness.

Methods: We extracted all individual actions from action plans developed by ICUs that received A\&F with $(n=10)$ and without $(n=11)$ toolbox for 6 months and classified them using Clinical Performance Feedback Intervention Theory. We held semi-structured interviews with participants during the trial. We compared the number and type of planned and completed actions between study groups and explored barriers and facilitators to effective action planning.

Results: ICUs with toolbox planned more actions directly aimed at improving practice $(p=0.037)$ and targeted a wider range of practice determinants compared to ICUs without toolbox. ICUs with toolbox also completed more actions during the study period, but not significantly $(p=0.142)$. ICUs without toolbox reported more difficulties in identifying what actions they could take. Regardless of the toolbox, all ICUs still experienced barriers relating to the feedback (low controllability, accuracy) and organisational context (competing priorities, resources, cost).

Conclusions: The toolbox helped health professionals to broaden their mindset about actions they could take to change clinical practice. Without the toolbox, professionals tended to focus more on feedback verification and exploring solutions without developing intentions for actual change. All feedback recipients experienced organisational barriers that inhibited eventual completion of actions.
\end{abstract}

Trial registration: ClinicalTrials.gov, NCT02922101. Registered on 26 September 2016.

Keywords: Intensive care, Medical audit, Feedback, Quality improvement, Quality indicators

\footnotetext{
* Correspondence: w.t.gude@amc.uva.nl

${ }^{+}$Wouter T. Gude and Marie-José Roos-Blom contributed equally to this work.

'Department of Medical Informatics, Amsterdam UMC, Amsterdam Public

Health Research Institute, University of Amsterdam, Amsterdam, The

Netherlands

Full list of author information is available at the end of the article
}

(c) The Author(s). 2019 Open Access This article is distributed under the terms of the Creative Commons Attribution 4.0 International License (http://creativecommons.org/licenses/by/4.0/), which permits unrestricted use, distribution, and reproduction in any medium, provided you give appropriate credit to the original author(s) and the source, provide a link to the Creative Commons license, and indicate if changes were made. The Creative Commons Public Domain Dedication waiver (http://creativecommons.org/publicdomain/zero/1.0/) applies to the data made available in this article, unless otherwise stated. 


\section{Contributions to the literature}

- Based on half a century of audit and feedback (A\&F) research, we know A\&F interventions are more effective if they facilitate action planning, but little is known about how best to do this. In a cluster RCT (published elsewhere), we showed that a toolbox that suggests improvement actions increased A\&F effectiveness.

- The current study focused on understanding the mechanism by which the toolbox increased A\&F effectiveness. We showed that feedback recipients who used the toolbox progressed through the action planning processes quicker, whilst targeting more areas for improvement.

- We also showed that end-users preferred toolbox actions targeting care processes over those targeting outcomes because the latter require more organisational and cultural changes.

- Our findings contribute to the design of action planning functionalities in A\&F interventions and underline which barriers could be overcome by an action implementation toolbox and which barriers would require additional co-interventions.

\section{Introduction}

Audit and feedback (A\&F) is an important strategy to close the gap between actual and desired clinical practice, but its observed effects vary greatly across studies [1]. The Cochrane review of 140 randomised controlled trials (RCTs) identified action planning to be an important effect modifier of A\&F [1]. However, few A\&F trials explicitly described action planning as part of the intervention, and those trials that did appeared to deliver this component of the intervention in various ways $[2,3]$. As a result, some recent studies that aimed to reproduce the positive effect of action planning have failed [4]. This indicates that the review's finding about the positive effect of action planning, derived from indirect comparisons from meta-regressions, is insufficiently reliable and does not inform us how to incorporate action planning as an intervention component to A\&F [5, 6]. Conducting head-to-head trials instead, comparing different action planning approaches in addition to providing $A \& F$, would provide useful information to guide the operationalisation of action planning in A\&F and other quality improvement interventions [7-9]. Although action planning is a familiar activity in clinical practice, health professionals often lack the time, skills or knowledge to interpret feedback and formulate and plan improvement actions [10]. Providing an action implementation toolbox, containing suggested actions and materials linked to specific potential barriers in the care process, may support this action planning process.
The Dutch National Intensive Care Evaluation (NICE) foundation developed an electronic A\&F intervention to improve pain management practice in Dutch ICUs $[11,12]$. In this context, optimal pain management means that pain is measured in every patient in each shift; pain scores are usually acceptable; and if they are not, appropriate treatment is given and pain measurement is repeated within $1 \mathrm{~h}$ to evidence that the pain is normalised. The intervention was evaluated in a head-to-head cluster RCT in which all participating ICUs received feedback on four pain management indicators. ICUs were randomised to receive a blank structured action plan with action implementation toolbox (feedback with toolbox group) and the other half to receive a blank structured action plan but no toolbox (feedback only group) [13]. The toolbox listed potential barriers in the pain management process alongside suggested actions and materials to address these (Fig. 1) [11]. Based on theory and previous empirical research $[6,14-16]$, we hypothesised that the toolbox would increase the effectiveness of the A\&F intervention by helping ICU teams to formulate and implement improvement actions. Consistent with this hypothesis, the trial analysis found absolute improvement on overall pain management performance in both the feedback with toolbox group (14.8\%; 95\% confidence interval [CI], 14.0-15.5) and the feedback only group (4.8\%; 95\% CI, 4.2-5.5), with the feedback with toolbox group achieving significantly larger effects than the feedback only group $(p=0.049)$ [13].

In this study, we aimed to understand the mechanisms through which the A\&F intervention in combination with the action implementation toolbox facilitated ICUs' action planning processes to inform future A\&F and toolbox research. To achieve this, we examined how action planning processes differed between the study arms in terms of the development of action plans, the extent to which those plans were successfully executed and what barriers and facilitators were experienced during these processes.

\section{Methods}

Study setting and participants

The study was carried out alongside a cluster RCT (ClinicalTrials.gov NCT02922101). Twenty-one ICUs in the Netherlands participated, including 25,141 patient admissions $[11,13]$. ICUs were eligible to participate if they (1) had allocated staff to set up a local quality improvement team, consisting of at least one intensivist and one nurse, and-in addition to their regular data upload to the NICE quality registry-(2) had the possibility to submit all data items required for calculating four newly defined pain management indicators [17]. Each ICU identified one team member as the primary contact for the research team during the trial. The medical manager of each ICU signed a consent form to formalise their commitment. ICUs started receiving the intervention between January 2017 and 


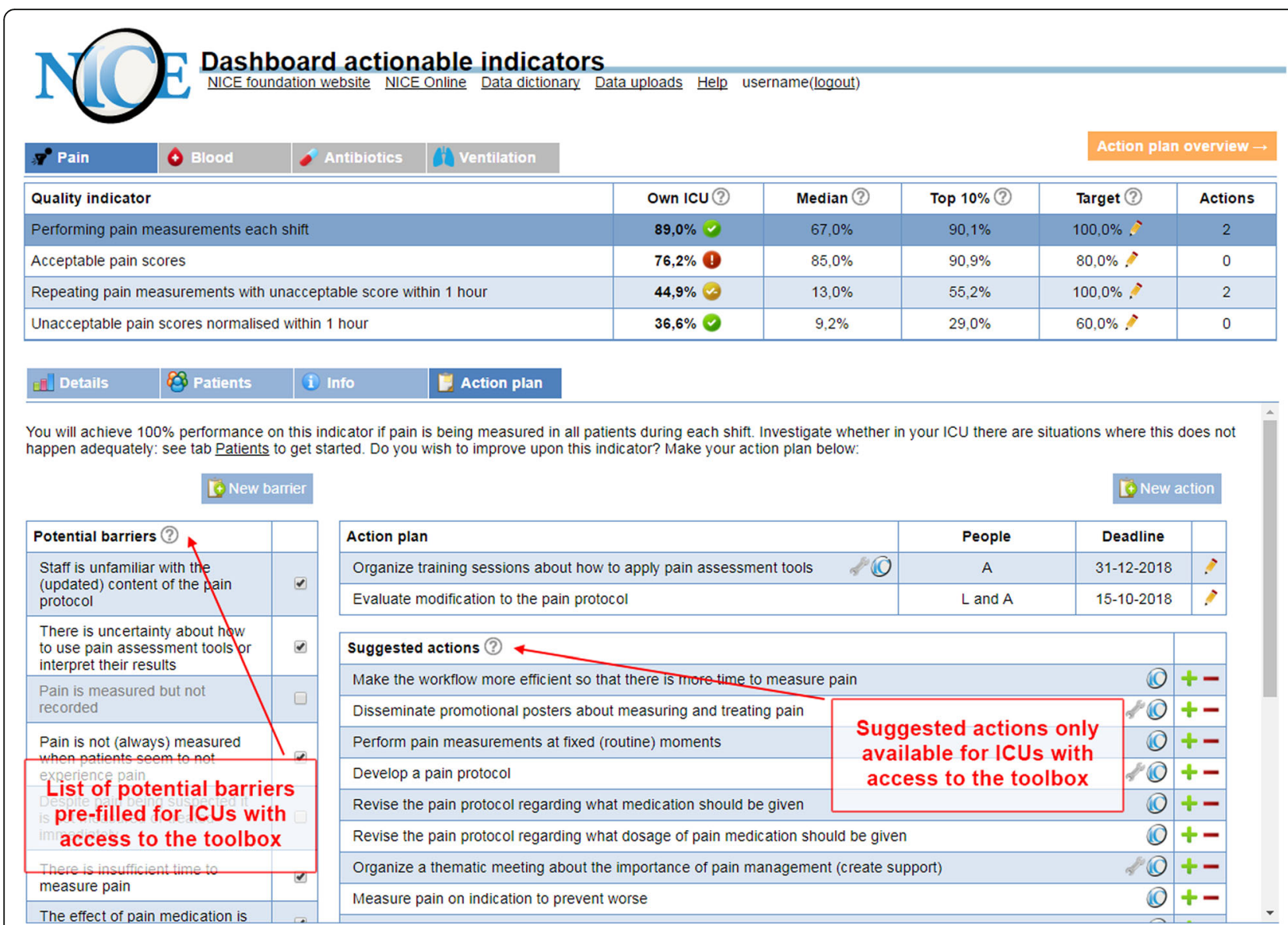

Fig. 1 The NICE dashboard displayed an overview of pain management performance (upper part) and four types of pages specific to the selected indicator (lower part). The difference between study groups was only in the action plan page. The feedback only group received a blank structured action plan and could record and update potential barriers and intended actions. The action plan for the feedback with toolbox group was supplemented with a pre-filled list of potential barriers and suggested actions (indicated by the NICE icon). Some actions included supporting materials (indicated by a wrench icon) available for download. Users could add suggested actions to their action plan and specify their description (plus sign) or hide them if they were not relevant (minus sign)

November 2017 for a duration of 6 months. ICUs were aware that there were two variations of the intervention being evaluated, but they were not told what aspect (i.e. the toolbox) was randomised. Figure 1 displays a screenshot of the dashboard and describes how action planning with and without action implementation toolbox took place.

\section{Theoretical framework}

The recently published Clinical Performance Feedback Intervention Theory (CP-FIT) provides information about how feedback works in practice and what factors may influence its effects [18]. It builds upon 30 preexisting theories relating to feedback and a meta-synthesis of 65 qualitative evaluation studies of 73 A\&F interventions. CP-FIT presents a feedback cycle consisting of a sequence of ten processes: Goal setting, Data collection and analysis, Feedback, recipient Interaction, Perception, optional Verification and Acceptance of the feedback, followed by Intention, Behaviour and Clinical performance improvement (see Fig. 2). It proposes that feedback becomes less effective if any of those individual processes fails, causing progress around the cycle to slow or stop (e.g. if feedback recipients do not accept the feedback, they will not develop improvement intentions). Feedback processes' success or failure is influenced by 70 factors relating to the feedback itself, the recipient of the feedback and the context in which the feedback is delivered. These factors exert their effects via seven explanatory mechanisms of increasing or decreasing the feedback's Complexity, Relative advantage, Resource match, Compatibility, Credibility, Social influence and Actionability [18].

For the current study, we were interested into what extent ICU teams confronted with feedback (Perception) react with planning (Intention) and completing (Behaviour) actions aimed at actually improving practice. Following CP-FIT, ICUs may also plan actions that may not directly lead to Clinical performance improvement, such 


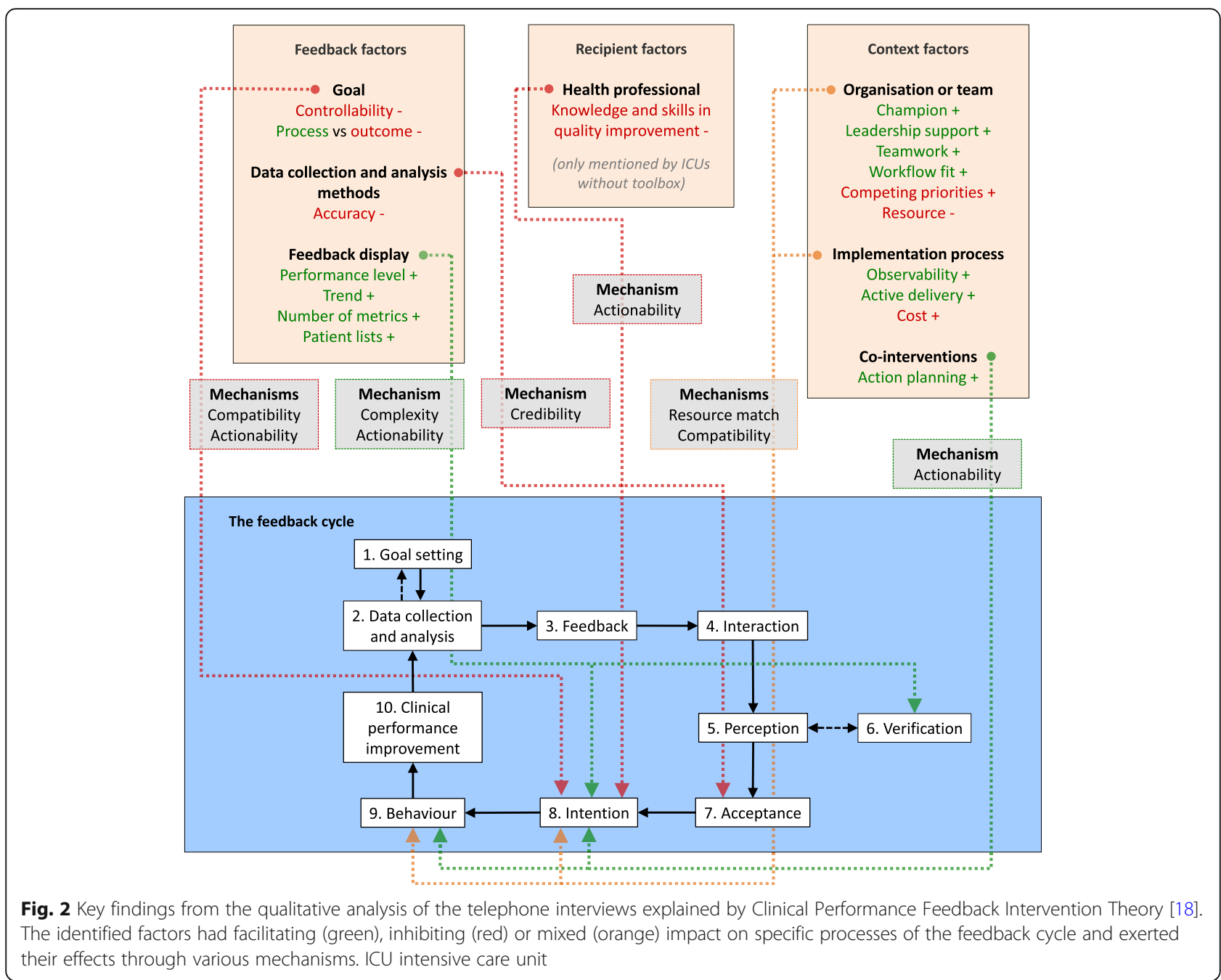

as verifying the feedback's underlying data (Verification, but no Acceptance) or exploring possible solutions to the problem (Acceptance, but no Intention).

\section{Data collection}

We extracted all individual actions from the action plans that ICUs had developed and maintained in the electronic dashboard as part of the intervention. This included the actions' titles, descriptions, deadlines, assigned people, status (in progress, completed, cancelled), whether they were self-defined or selected from the toolbox and timestamps of any action plan changes (e.g. status update).

We held brief semi-structured telephone interviews with the ICUs' main contact every 4-6 weeks during the trial to explore which barriers and facilitators ICUs experienced relating to the action planning process. We also verified whether action plans were up-to-date and encouraged ICUs to update the action plan if this was not the case. The structure of the calls was the same for ICUs in both study groups, with the exception that the action implementation toolbox and its contents were not mentioned to ICUs in the feedback only group. The interview topic guide is included as Additional file 1. The interviews were conducted by one researcher (WG or MRB) and lasted approximately 10-15 min. Interview notes were emailed to the ICU team for member checking [19].

\section{Analysis}

Two researchers (WG and MRB, who also conducted the interviews) independently coded all actions from the action plans in three steps as described below; discrepancies were resolved through consensus. First, we labelled the individual actions to identify similar actions. We derived labels from the list of toolbox actions as a starting point and iteratively added labels for self-defined actions that did not match any of the pre-defined toolbox actions. Second, we used CPFIT to identify for each action which feedback cycle process it reflected (i.e. Verification, Acceptance, Intention or Behaviour) to indicate its proximity to actual practice change. Third, we grouped those actions reflecting Intention or 
Behaviour using the integrated framework for identifying factors that prevent or enable improvements in healthcare professional practice by Flottorp et al. [20] to understand which practice determinants ICUs had targeted for change. Practice determinants are "factors that might prevent or enable improvements in that practice" and have been organised in seven domains: guideline factors (e.g. compatibility of the recommended behaviour), individual health professional factors (e.g. awareness with the recommendation), patient factors (e.g. patient preferences), professional interactions (e.g. communication and influence), incentives and resources (e.g. information system), capacity for organisational change (e.g. leadership) and social, political or legal factors (e.g. payer or funder policies) [20].

We counted the number of actions per feedback cycle process [18] and per targeted practice domain [20] for both study groups. We used chi-square tests to test whether the distribution of actions across the feedback cycle processes and targeted practice domains differed between groups. We also calculated ICUs' median and interquartile range (IQR) number of planned and completed actions, completion rates and time (in days) taken to complete actions; we tested the difference between groups using the Wilcoxon test. We also counted for each unique action the number of ICUs in both study groups that had planned and/or completed the action during the trial. This allowed us to assess the added value of the toolbox by understanding (1) which actions were most or least often selected, (2) which actions were easy or difficult to complete and (3) to what extent and how toolbox actions differed from self-defined actions.
The interview transcripts were independently coded by two researchers (WG and MRB) using CP-FIT's codebook [18]. For each barrier or facilitator mentioned by ICUs, we marked which mechanisms they activated and which feedback cycle process of interest (i.e. Verification, Acceptance, Intention or Behaviour) was affected.

\section{Results}

Table 1 displays the baseline characteristics of the 21 ICUs that participated in the trial (10 in the feedback with toolbox group and 11 in the feedback only group). ICU teams usually consisted of three to five members and included intensivists, nurses and managers. The median baseline pain management performance was, despite randomisation, significantly lower in the feedback with toolbox group compared to the feedback only group (70.2\% vs. $62.5 \% ; p=0.049)$ [13].

\section{Action plans}

Action plans contained a total of 234 individual actions (upper part Table 2). Fifty-three (34.6\%) actions reflected the Verification or Acceptance process of the feedback cycle, with the remaining 181 (77.4\%) targeting actual practice change. Sixty-five (27.8\%) of these were still in progress, reflecting Intention, and 116 (49.6\%) were completed, reflecting Behaviour. The distribution of the actions' coded positions in the feedback cycle differed significantly between study groups $(p=0.012)$, with $87.3 \%$ (128 out of 153) of the actions in the feedback with toolbox group reflecting the Intention or Behaviour process compared to $65.4 \%$ (53 out of 81 ) in the feedback only group.

Table 1 Characteristics of the 21 ICUs participating in the cluster-randomised controlled trial

\begin{tabular}{lll}
\hline ICU characteristic & Feedback with toolbox $(n=10)$ & Feedback only $(n=11)$ \\
\hline Hospital type, $n$ (\%) & & $2(18 \%)$ \\
Academic & $1(10 \%)$ & $9(72 \%)$ \\
$\quad$ Non-academic & $9(90 \%)$ & $16.0(11.0-24.0)$ \\
Number of beds, median (IQR) & $12.5(9.0-15.5)$ & $1048(757-2046)$ \\
Number of admissions per year, median (IQR) & $835(645-1726)$ & $423(223-1167)$ \\
$\quad$ Surgical admissions & $288(215-1051)$ & $668(524-774)$ \\
Medical admissions & $533(313-697)$ & $4(3-4)$ \\
Number of QI team members, median (IQR) & $4(3-5)$ & $70.2(53.3-76.0)$ \\
Baseline pain management performance*, median (IQR) & $62.5(51.6-76.1)$ & $81.4(58.4-87.8)$ \\
Indicator 1-measuring pain each shift & $67.8(61.8-82.3)$ & $85.3(82.7-87.7)$ \\
Indicator 2-acceptable pain scores & $87.5(80.6-91.3)$ & $13.0(8.4-19.3)$ \\
Indicator 3-repeating pain measurements within 1 h & $14.4(5.2-28.7)$ & $9.6(6.8-15.7)$ \\
Indicator 4-normalised pain scores within 1 h & $12.3(4.1-22.6)$ & \\
\hline
\end{tabular}

ICU intensive care unit, IQR interquartile range, Q/ quality improvement

*The performance composite was measured over the preceding 3 months and defined as the percentage of patient-shift observations during which pain was measured at least once and no unacceptable pain score was observed and/or unacceptable pain scores were followed by a repeated measurement and normalised within $1 \mathrm{~h}$ 
Table 2 Comparison of the action plan contents between the feedback with toolbox (10 ICUs) and feedback only (11 ICUs) group

\begin{tabular}{|c|c|c|c|}
\hline Action plan contents (all actions) & Feedback with toolbox $(n=153)$ & Feedback only $(n=81)$ & $p$ value \\
\hline Position in the feedback cycle* & & & 0.012 \\
\hline Verification (e.g. check data accuracy, understand the problem) & $9(5.9)$ & $10(12.3)$ & \\
\hline Acceptance (e.g. explore possible solutions) & $16(10.5)$ & $18(22.2)$ & \\
\hline Intention (i.e. active effort to change practice) & $49(32.0)$ & $16(19.8)$ & \\
\hline Behaviour (i.e. practice change) & $79(51.6)$ & $37(45.7)$ & \\
\hline Total number of actions completed & $96(62.7)$ & $51(63.0)$ & \\
\hline Action plan contents (actions reflecting Intention or Behaviour to change practice) & Feedback with toolbox $(n=128)$ & Feedback only $(n=53)$ & $p$ value \\
\hline \multicolumn{4}{|l|}{ Origin } \\
\hline Selected from the toolbox & $104(81.3)$ & & \\
\hline Self-defined; similar action available in the toolbox & $13(10.2)$ & $32(60.4)$ & \\
\hline Self-defined; no similar action available in the toolbox & $11(8.6)$ & $21(39.6)$ & \\
\hline Practice domain targeted* & & & 0.331 \\
\hline Guideline & $21(16.4)$ & $6(11.3)$ & \\
\hline Individual health professional & $50(39.1)$ & $29(54.7)$ & \\
\hline Professional interactions & $3(2.3)$ & $1(1.9)$ & \\
\hline Incentives and resources & $36(28.1)$ & $14(26.4)$ & \\
\hline Capacity for organisational change & $13(10.2)$ & $3(5.7)$ & \\
\hline Patient & $5(3.9)$ & $0(0.0)$ & \\
\hline Total number of actions completed & $79(61.7)$ & $37(69.8)$ & \\
\hline Median number of actions planned (IQR) & $8(6.25-14.75)$ & $5(2.5-5)$ & 0.037 \\
\hline Median number of actions completed (IQR) & $4(2-11)$ & $3(1-3)$ & 0.142 \\
\hline Median percentage of actions completed (IQR) & $59.2(37.9-94.1)$ & $60(20-75)$ & 0.479 \\
\hline Median average number of days taken to complete actions (IQR) & $56.5(32.6-125.4)$ & $59.8(44-95.8)$ & 0.762 \\
\hline
\end{tabular}

Considering only the subset of those actions reflecting Intention or Behaviour to change practice (lower part Table 2), ICUs in the feedback with toolbox group planned a median of 8 (6.25-14.75) actions and completed 4 (IQR, $2-11)$ actions during the intervention period. They picked 104 (81.3\%) of 128 actions from the toolbox whilst the remaining 24 were self-defined. ICUs in the feedback only group planned a median of 5 (IQR, 2.5-5) actions and completed 3 (IQR, 1-3); 32 (60.4\%) of the 53 actions in the group were equal or similar to actions present in the toolbox. Whereas the median number of planned actions was significantly higher in the feedback with toolbox group $(p=0.037)$, this was not the case for the median number of completed actions $(p=0.142)$. In both groups, ICUs completed approximately $60 \%$ of their planned actions during the intervention period $(p=0.479)$; each action took them on average 2 months to complete $(p=0.762)$.

In both study groups, actions most often sought practice change in individual health professional factors (39.1\% and 54.7\%) and resources (28.1\% and 26.4\%) based on Flottorp et al.'s integrated framework for identifying factors that prevent or enable improvements in healthcare professional practice [20]. The distribution of the actions across targeted practice domains was similar between the two groups $(p=0.331)$ (Table 2). We identified 29 unique actions from the action plans of both study groups (Table 3). Action plans from ICUs in the feedback with toolbox group included 26 (89.7\%) of these and the feedback only group included 19 (65.5\%) of these. Ten unique self-defined actions did not match any of the toolbox actions (indicated with an asterisk in Table 3). Two toolbox actions (keep standard prescriptions of pain medication readily available; increase nurses' autonomy to prescribe pain medication) were not planned by any ICU during the trial.

\section{Experienced barriers and facilitators to action planning processes in the feedback cycle}

From the interviews, we identified 19 factors (italicised in brackets) that emerged as barriers or facilitators to action planning processes. These factors exerted their effects through five mechanisms (italicised) onto 
Table 3 Unique actions $(n=29)$ grouped by practice domain with the percentage of ICUs that planned (and completed) these actions

\begin{tabular}{|c|c|c|}
\hline \multirow[t]{2}{*}{ Unique actions grouped by practice domain } & \multicolumn{2}{|c|}{$\%$ of ICUs that planned (completed) the action } \\
\hline & Feedback with toolbox $(n=10)$ & Feedback only $(n=11)$ \\
\hline Guideline (e.g. clarity of the recommendation, accessibility) & $60(40)$ & $36.4(27.3)$ \\
\hline Revise pain protocol about pain monitoring (e.g. frequency, triggers) & $40(20)$ & $9.1(0)$ \\
\hline Workflow redesign; introduce routine to measuring pain & $20(20)$ & $9.1(9.1)$ \\
\hline Increase protocol accessibility (e.g. electronically, flow chart, pocket card) & $20(10)$ & $9.1(9.1)$ \\
\hline Develop pain protocol & $20(10)$ & - \\
\hline Revise pain protocol about pain medication (e.g. drug choice, dosage choice) & $10(10)$ & $27.3(18.2)$ \\
\hline Workflow redesign; check contraindications to pain medications at admission & $10(0)$ & - \\
\hline Individual health professional (e.g. knowledge and skills; cognitions) & $90(70)$ & $81.8(63.6)$ \\
\hline Digital newsletter or email & $70(60)$ & $27.3(27.3)$ \\
\hline Educational meeting & $70(50)$ & $9.1(9.1)$ \\
\hline Individual feedback if pain has not been measured & $60(20)$ & $9.1(9.1)$ \\
\hline Promotional poster or message board & $40(30)$ & $36.4(27.3)$ \\
\hline Announcement about pain monitoring during regular staff meeting* & $10(0)$ & $72.7(63.6)$ \\
\hline Quiz about ICU pain management knowledge* & - & $9.1(9.1)$ \\
\hline Professional interactions (e.g. communication and influence; team processes) & $30(10)$ & $9.1(9.1)$ \\
\hline Include information on pain status to hand-over moments during shift change & $20(0)$ & $9.1(9.1)$ \\
\hline Collaborate with Acute Pain Service about pain medication after surgery* & $10(10)$ & - \\
\hline Incentives and resources (e.g. availability of resources; information system) & $100(80)$ & $63.6(36.4)$ \\
\hline Build or adapt reminder system in the EHR & $80(50)$ & $36.4(18.2)$ \\
\hline Increase completeness of pain measurement data recording in the local EHR & $50(30)$ & - \\
\hline Expand order set in EHR (e.g. add pain measurement order at admission)* & $40(40)$ & $18.2(9.1)$ \\
\hline Provide validated pain measurement tools (i.e. NRS, VAS, BPS, CPOT) & $30(20)$ & - \\
\hline Adapt EHR form to facilitate pain measurement recording* & $10(10)$ & $18.2(9.1)$ \\
\hline Create performance monitoring tool/dashboard* & $10(0)$ & $9.1(0)$ \\
\hline Nonfinancial incentives (e.g. edible treat) if targets are achieved* & - & $9.1(9.1)$ \\
\hline Keep patient-controlled analgesia (PCA) pumps in stock locally at ICU* & - & $9.1(0)$ \\
\hline Capacity for organisational change (e.g. mandate, authority, accountability) & $40(40)$ & $27.3(18.2)$ \\
\hline Assign or involve a pain coordinator & $40(40)$ & $18.2(9.1)$ \\
\hline Workflow redesign; increase efficiency to allow more time for measuring pain & $10(10)$ & - \\
\hline Expand pain management Ql team* & $10(10)$ & - \\
\hline Monitoring and feedback on (recording of) pain measurements each shift* & $10(0)$ & $9.1(9.1)$ \\
\hline Monitoring and feedback on appropriateness of prescribed pain medication & $10(0)$ & - \\
\hline Patient (e.g. patient needs; preferences) & $30(10)$ & - \\
\hline Workflow redesign; monitor/manage factors (e.g. fear) that may worsen pain & $20(0)$ & - \\
\hline Workflow redesign; measure pain on patients' indication & $10(10)$ & - \\
\hline
\end{tabular}

${ }^{*}$ A self-defined action that did not match any toolbox action

multiple processes in the feedback cycle. Figure 2 displays a schematic overview.

\section{Feedback factors}

ICUs in both groups felt that the goal of the feedback, adequate pain management, was not always under their control (Controllability), e.g. when patients were admitted with unacceptable pain from other departments. Additionally, ICUs found it easier to remind and encourage staff to measure pain more frequently than to make changes to pain medication to prevent or normalise unacceptable pain scores (Process vs outcome). These factors 
inhibited ICUs' improvement intentions by decreasing ICUs' Compatibility with the feedback, and the feedback's Actionability.

The data collection and analysis methods were questioned by some ICUs as they suspected missing pain measurements in the data underlying the feedback, differences in how pain was determined and mismatching shift times (Accuracy). This hampered the feedback's Credibility and inhibited ICUs' acceptance of the feedback.

Low-performance scores on indicators (Performance level) in the dashboard facilitated ICUs' intentions by increasing Actionability. The breakdown of performance in a number of relevant subgroups (Number of metrics) and patient lists (Patient lists) helped ICUs to verify the feedback by reducing Complexity and facilitated intention to improve by making it clear how to focus their actions, increasing Actionability. For example, subgroup analyses often showed that pain was more prevalent amongst surgical admissions, and pain measurements were often not performed during a patient's first shift after admission. Also, ICUs reported that the performance charts (Trend) reduced the feedback's Complexity by supporting the interpretation of the effects of actions and decisions on whether their action plan needed amending.

\section{Recipient factors}

Particularly ICUs in the feedback only group reported running out of ideas on what actions to take (Knowledge and skills in quality improvement), which reduced the Actionability and inhibited improvement intentions. Multiple of these ICUs expressed an interest in learning from best practices in high performing ICUs. Conversely, ICUs in the feedback with toolbox group mentioned the toolbox gave them good suggestions for improvement.

\section{Context factors}

With regard to organisational and team factors, many ICUs had one or two people responsible for implementing the intervention locally (Champion). They would typically review the feedback in the dashboard, discuss it with the wider team and update their action plan accordingly (Teamwork). ICUs mentioned having a manager as an active member of the local QI team (Leadership support) as a facilitating factor. Action planning processes were more efficient in ICUs that already had some QI structures in place before the intervention, including a dedicated QI team and structural meetings (Workflow fit), which increased Resource match and Compatibility. In contrast, all ICUs reported experiencing barriers relating to other tasks, events and busy clinical practice (Competing priorities), such as the implementation of a new EHR. Limited resources in terms of staff and time (Resources) led to actions being delayed or cancelled.

Factors relating to the implementation process exerted their effects through the same mechanisms of Resource match and Compatibility. ICUs noticed the positive effects of their completed actions (Observability), both via the dashboard's charts and in clinical interactions, which motivated them to continue with their action planning processes. At the same time, observability was hampered by pain measurements being performed but not recorded in the EHR. Recording all pain measurements in the EHR was considered too resource intensive (Cost), especially if patients did not seem to be in pain. The telephone interviews (Active delivery) stimulated most ICUs to keep reviewing their feedback, reinforce actions that had stagnated, and update their action plans.

The dashboard's structured action plan facilitated ICUs' action planning processes (Action plan). This was particularly mentioned by ICUs in the feedback with toolbox group. Overall, the toolbox was considered to provide useful guidance for improvement: a team member would typically go through the list of suggested actions and pick those that could be beneficial and feasible for their own local practice. A number of suggestions were deemed too generic, simple or inapplicable, but were often still sufficient as a starting point for ICUs to formulate actions more tailored to their local needs.

\section{Discussion}

Our study revealed that ICUs with access to an action implementation toolbox planned more actions to improve practice and targeted a wider range of practice domains than ICUs without toolbox. ICUs without toolbox tended to remain longer in earlier steps of the feedback cycle such as verifications of the feedback data and explorations of possible solutions, rather than targeting actual practice change. However, although ICUs with toolbox also completed more actions during the study period, this difference was not significant. The toolbox facilitated ICUs' action planning processes by increasing the actionability because it filled a knowledge gap for health professionals; ICUs in the feedback only group reported more difficulties in identifying what actions they could take. However, all ICUs still experienced barriers in the feedback (low controllability and accuracy) and context (competing priorities, limited time and staff resources and cost) that were not targeted by the toolbox or other components of the A\&F intervention.

ICUs without toolbox typically focused on low effort activities to increasing staff awareness and knowledge using existent structures (e.g. announcements during regular staff meetings) compared to ICUs with toolbox and expressed that they eventually ran out of ideas on what actions to take. This resonates with various studies 
that have demonstrated that A\&F recipients often find it difficult to formulate improvement actions [10, 21-23]. In several previous A\&F studies in ICU settings, health professionals have experienced the $\mathrm{A} \& \mathrm{~F}$ as insufficiently actionable [24, 25]. In our study, the toolbox increased actionability, leading to more improvement intentions. Furthermore, toolbox ICUs were able to go beyond increasing staff awareness and knowledge to changing existing workflows.

The lack of difference in the number of completed actions may indicate that the toolbox had limited impact on the extent to which improvement intentions were translated into actual practice change (i.e. behaviour). The related organisational barriers that ICUs experienced have been frequently identified in other studies [26]. Since intentions to change practice are meaningless if they cannot be translated into action, more efforts are needed to facilitate health professionals in this translation. Most of ICUs' actions targeted care processes (i.e. increasing the frequency of pain measurements) rather than outcomes (i.e. reducing pain). It is a common finding that processes are more commonly targeted than outcomes [27], which often relates to their actionability and controllability, yet those processes may have only an indirect link to improving patient outcomes. Some of the toolbox actions to specifically improve outcomes were hardly or never selected by ICUs, such as increasing nurses' autonomy to prescribe pain medication. In this setting, such actions may not have been selected due to their invasiveness, consequences for the organisational context and inertia to change traditional divisions of labour (that is, pain medication is prescribed by intensivists, whilst nurses are responsible for monitoring pain and administering the drugs) $[28,29]$.

\section{Implications for practice and research}

Our findings imply that providing recipients of $A \& F$ interventions with practical suggestions for improvement actions is a promising approach to increasing the effectiveness of A\&F by making it more actionable. Feedback actionability is known to substantially contribute to the success of interventions [30,31], but has proven difficult to operationalise in practice $[4,6,24,25]$. We found that presenting suggested actions for improvement in a toolbox integrated within an electronic, structured action plan is a feasible and potentially less resource-intensive alternative to, for example, educational outreach visits [32]. Some of the remaining barriers to the action planning we identified (e.g. controllability) could be overcome by improving the toolbox contents, whereas others (particularly those relating to the organisational context) may require other co-interventions.

ICUs selected a wide variety of toolbox actions and added a range of self-defined actions that did not match any of the pre-defined toolbox actions. This confirms that different A\&F recipients may have different quality improvement needs [33]. We recommend that in the future A\&F interventions with action toolbox should, similar to the toolbox in this study, enable recipients to further tailor the toolbox to local context by adding self-defined actions and hide toolbox actions or amend their description in order to tailor the toolbox to their local context.

We derived the toolbox content from guidelines, literature and expert opinion [11, 12]. Although participants considered this useful, they expressed an additional interest in best practices from high performers. Future A\&F studies could therefore consider adding "best practicebased" actions to complement toolbox actions derived from other sources and dynamically update the toolbox by further refining actions with low completion rates.

\section{Strengths and limitations}

Our study has several strengths. Whereas the head-tohead RCT had previously demonstrated that the toolbox was effective in improving ICU pain management [13], the current study helped us understand the underlying mechanisms for how it was effective. This contributes to expanding our knowledge about how action planning in A\&F interventions can be facilitated [7]. The electronic nature of our A\&F intervention enabled us to study mechanisms in detail and unobtrusively, whilst including all participants [34, 35]. Usage data that are a by-product of using digital $\mathrm{A} \& \mathrm{~F}$ interventions in real-world settings is largely underutilised; they also informed the telephone interviews. This created a synergistic mixed-methods approach in which quantitative and qualitative complemented as well as strengthened each other [36].

Some limitations warrant discussion. The toolbox made it easier for ICUs to select (many) actions by one click of the mouse, whilst ICUs without toolbox had to formulate actions themselves. In addition, self-defined actions often differed in "load" and potential impact. This hampered a straightforward comparison on the number of actions between groups. We addressed this by harmonising individual actions based on their content during the first step of our coding procedure. Furthermore, telephone interviews often prompted ICUs to update their plans. As a result, the recorded number of days needed to complete actions is likely overestimated. Next, we did not assess which suggested actions from the toolbox were already implemented in practice before the start of the intervention, which limited our interpretation of why particular actions were not selected by ICUs. Finally, the contents of the toolbox were developed specifically for Dutch ICU setting, and the feasibility of the toolbox' suggested actions may not be generalisable to other settings. 


\section{Conclusion}

An action implementation toolbox containing suggested actions for practice change increases the actionability of A\&F interventions, prompting recipients to initiate more changes and across a wider range of practice determinants. The toolbox helped health professionals to broaden their mindset about actions they could take to improve practice. Without the toolbox, professionals tended to focus more on feedback verification and exploring solutions without developing intentions for actual change. All feedback recipients experienced barriers that inhibited completion of actions. Whereas some of those barriers may be overcome when appropriately addressed by a toolbox, other barriers, often relating to the organisational context, will require co-interventions.

\section{Additional file}

Additional file 1: Standards for Reporting Implementation Studies: the StaRI checklist for completion. (DOCX $78 \mathrm{~kb}$ )

\section{Abbreviations}

A\&F: Audit and feedback; CRCT: Cluster-randomised controlled trial; ICU: Intensive care unit; IQR: Interquartile range; NICE: National Intensive Care Evaluation foundation; SD: Standard deviation

\section{Acknowledgements}

The authors would like to thank our software engineers Richard Minne, Eric van der Zwan and Winston Tjon Sjoe Sjoe for their work in developing the NICE dashboard.

\section{Authors' contributions}

WG, MRB, SvdV, DD, EdJ, NP and NdK contributed to the study conception and participated in critically appraising and revising the intellectual content of the manuscript. WG and MRB were primarily responsible for the data collection and analysis and the manuscript draft. All authors read and approved the final manuscript.

\section{Funding}

This study was funded by the National Intensive Care Evaluation (NICE) foundation's own resources. SvdV is supported by the Health e-Research Centre (MRC research grant no. MR/K006665/1).

\section{Availability of data and materials}

The datasets used and/or analysed during the current study are available from the corresponding author on reasonable request.

\section{Ethics approval and consent to participate}

The Institutional Review Board (IRB) of the Academic Medical Center (Amsterdam, The Netherlands) informed us that formal IRB approval and patient consent were not deemed necessary because to the focus of intervention on improving organisational processes; individual patients will not be directly involved (IRB reference number: W16_271). Additionally, in the Netherlands, there is no need to obtain consent to use data from registries that do not contain patient-identifying information, as is the case in the NICE registry. The NICE foundation is officially registered according to the Dutch Personal Data Protection Act.

\section{Consent for publication}

Not applicable.

\section{Competing interests}

The authors declare that they have no competing interests.

\section{Author details}

${ }^{1}$ Department of Medical Informatics, Amsterdam UMC, Amsterdam Public Health Research Institute, University of Amsterdam, Amsterdam, The Netherlands. ${ }^{2}$ Department of Intensive Care Medicine, Amsterdam UMC, University of Amsterdam, Amsterdam, The Netherlands. ${ }^{3}$ National Intensive Care Evaluation (NICE) Foundation, Amsterdam, The Netherlands. ${ }^{4}$ Centre for Health Informatics, Division of Informatics, Imaging and Data Sciences, Faculty of Biology, Medicine and Health, Manchester Academic Health Science Centre, The University of Manchester, Manchester, UK. ${ }^{5}$ Department of Intensive Care Medicine, Leiden University Medical Center, Leiden, The Netherlands. ${ }^{6}$ NIHR Greater Manchester Primary Care Patient Safety

Translational Research Centre, Manchester Academic Health Science Centre, The University of Manchester, Manchester, UK.

Received: 1 June 2019 Accepted: 27 August 2019

Published online: 18 September 2019

\section{References}

1. Ivers N, Jamtvedt G, Flottorp S, Young JM, Odgaard-Jensen J, French SD, et al. Audit and feedback: effects on professional practice and healthcare outcomes. Cochrane Database Syst Rev. 2012;6:CD000259. https://doi.org/ 10.1002/14651858.CD000259.pub3.

2. No authors listed. Medical audit in general practice. I: Effects on doctors' clinical behaviour for common childhood conditions. North of England Study of Standards and Performance in General Practice. BMJ 1992;304:1480-1484.

3. Gardner B, Whittington C, McAteer J, Eccles MP, Michie S. Using theory to synthesise evidence from behaviour change interventions: the example of audit and feedback. Soc Sci Med. 2010;70:1618-25. https://doi.org/10.1016/j. socscimed.2010.01.039.

4. Ivers NM, Tu K, Young J, Francis JJ, Barnsley J, Shah BR, et al. Feedback GAP: pragmatic, cluster-randomized trial of goal setting and action plans to increase the effectiveness of audit and feedback interventions in primary care. Implement Sci. 2013;8:142. https://doi.org/10.1186/1748-5908-8-142.

5. Ivers NM, Grimshaw JM, Jamtvedt G, Flottorp S, O'Brien MA, French SD, et al. Growing literature, stagnant science? Systematic review, meta-regression and cumulative analysis of audit and feedback interventions in health care. J Gen Intern Med. 2014;29:1534-41. https:// doi.org/10.1007/s11606-014-2913-y.

6. Gude WT, van Engen-Verheul MM, van der Veer SN, Kemps HMC, Jaspers MWM, de Keizer NF, et al. Effect of a web-based audit and feedback intervention with outreach visits on the clinical performance of multidisciplinary teams: a cluster-randomized trial in cardiac rehabilitation. Implement Sci. 2016;11:160. https://doi.org/10.1186/s13012-016-0516-1.

7. Ivers NM, Sales A, Colquhoun H, Michie S, Foy R, Francis JJ, et al. No more "business as usual" with audit and feedback interventions: towards an agenda for a reinvigorated intervention. Implement Sci. 2014;9:14. https:// doi.org/10.1186/1748-5908-9-14.

8. Ivers NM, Grimshaw JM. Reducing research waste with implementation laboratories. Lancet (London, England). 2016;388:547-8. https://doi.org/10. 1016/S0140-6736(16)31256-9.

9. Colquhoun HL, Carroll K, Eva KW, Grimshaw JM, Ivers N, Michie S, et al. Advancing the literature on designing audit and feedback interventions: identifying theory-informed hypotheses. Implement Sci. 2017;12:117. https:// doi.org/10.1186/s13012-017-0646-0.

10. Ivers N, Barnsley J, Upshur R, Tu K, Shah B, Grimshaw J, et al. My approach to this job is. One person at a time: perceived discordance between population-level quality targets and patient-Centred care. Can Fam Physician. 2014;60:258-66.

11. Gude WT, Roos-Blom MJ, van der Veer SN, de Jonge E, Peek N, Dongelmans DA, et al. Electronic audit and feedback intervention with action implementation toolbox to improve pain management in intensive care: protocol for a laboratory experiment and cluster randomised trial. Implement Sci. 2017;12:68. https://doi.org/10.1186/s13012-017-0594-8.

12. Roos-Blom M-J, Gude WT, Spijkstra JJ, de Jonge E, Dongelmans D, de Keizer NF. Measuring quality indicators to improve pain management in critically ill patients. J Crit Care. 2018;49:136-42. https://doi.org/10.1016/j.jcrc.2018.10.027.

13. Roos-Blom MJ, Gude WT, de Jonge E, Spijkstra JJ, van der Veer SN, Peek N, Dongelmans DA, de Keizer NF. Impact of audit and feedback with action implementation toolbox on improving ICU pain management: cluster- 
randomised controlled trial. BMJ Qual Saf. 2019. https:/doi.org/10.1136/bmjqs2019-009588. PMID: 31263017.

14. Carver CS, Scheier MF. Control theory: a useful conceptual framework for personality-social, clinical, and health psychology. Psychol Bull. 1982; 92:111-35. https://doi.org/10.1037/0033-2909.92.1.111.

15. Brehaut JC, Colquhoun HL, Eva KW, Carroll K, Sales A, Michie S, et al. Practice feedback interventions: 15 suggestions for optimizing effectiveness. Ann Intern Med. 2016;164:435-41. https://doi.org/10.7326/M15-2248.

16. Gude WT, Van Engen-Verheul MM, Van Der Veer SN, De Keizer NF, Peek $\mathrm{N}$. How does audit and feedback influence intentions of health professionals to improve practice? A laboratory experiment and field study in cardiac rehabilitation. BMJ Qual Saf. 2017;26:279-87. https://doi. org/10.1136/bmjqs-2015-004795

17. van de Klundert N, Holman R, Dongelmans DA, de Keizer NF. Data resource profile: the Dutch National Intensive Care Evaluation (NICE) registry of admissions to adult intensive care units. Int J Epidemiol. 2015;44:1850-1850h. https://doi.org/10.1093/ije/dyv291.

18. Brown B, Gude WT, Blakeman T, van der Veer SN, Ivers N, Francis JJ, et al. Clinical Performance Feedback Intervention Theory (CP-FIT): a new theory for designing, implementing, and evaluating feedback in health care based on a systematic review and meta-synthesis of qualitative research. Implement Sci. 2019;14:40. https://doi.org/10.1186/s13012-019-0883-5.

19. Birt L, Scott S, Cavers D, Campbell C, Walter F. Member checking: a tool to enhance trustworthiness or merely a nod to validation? Qual Health Res. 2016;26:1802-11. https://doi.org/10.1177/1049732316654870.

20. Flottorp SA, Oxman AD, Krause J, Musila NR, Wensing M, Godycki-Cwirko M, et al. A checklist for identifying determinants of practice: a systematic review and synthesis of frameworks and taxonomies of factors that prevent or enable improvements in healthcare professional practice. Implement Sci. 2013;8:35. https://doi.org/10.1186/1748-5908-8-35.

21. Morrell C, Harvey G, Kitson A. Practitioner based quality improvement: a review of the Royal College of Nursing's dynamic standard setting system. Qual Health Care. 1997;6:29-34.

22. Grant AM, Guthrie B, Dreischulte T. Developing a complex intervention to improve prescribing safety in primary care: mixed methods feasibility and optimisation pilot study. BMJ Open. 2014;4:e004153. https://doi.org/10.1136/ bmjopen-2013-004153.

23. Tierney S, Kislov R, Deaton C. A qualitative study of a primary-care based intervention to improve the management of patients with heart failure: the dynamic relationship between facilitation and context. BMC Fam Pract. 2014;15:153. https://doi.org/10.1186/1471-2296-15-153.

24. Sinuff T, Muscedere J, Rozmovits L, Dale CM, Scales DC. A qualitative study of the variable effects of audit and feedback in the ICU. BMJ Qual Saf. 2015; 24:393-9. https://doi.org/10.1136/bmjqs-2015-003978.

25. De Vos MLG, Van Der Veer SN, Graafmans WC, De Keizer NF, Jager KJ, Westert GP, et al. Process evaluation of a tailored multifaceted feedback program to improve the quality of intensive care by using quality indicators BMJ Qual Saf. 2013;22:233-41. https://doi.org/10.1136/bmjqs-2012-001375.

26. Cabana MD, Rand CS, Powe NR, Wu AW, Wilson MH, Abboud P-AC, et al. Why don't physicians follow clinical practice guidelines? J Am Med Assoc. 1999;282:1458. https://doi.org/10.1001/jama.282.15.1458.

27. Lilford R, Mohammed MA, Spiegelhalter D, Thomson R. Use and misuse of process and outcome data in managing performance of acute medical care: avoiding institutional stigma. Lancet. 2004;363:1147-54. https:/doi.org/ 10.1016/S0140-6736(04)15901-1.

28. Guidet B, González-Romá V. Climate and cultural aspects in intensive care units. Crit Care. 2011;15:312. https://doi.org/10.1186/cc10361.

29. van der Sluijs AF, van Slobbe-Bijlsma ER, Chick SE, Vroom MB, Dongelmans DA, Vlaar APJ. The impact of changes in intensive care organization on patient outcome and cost-effectiveness - a narrative review. J Intensive Care. 2017;5:13. https://doi.org/10.1186/s40560-016-0207-7.

30. Hysong SJ, Best RG, Pugh JA. Audit and feedback and clinical practice guideline adherence: making feedback actionable. Implement Sci. 2006;1:9. https://doi.org/10.1186/1748-5908-1-9.

31. Hysong SJ. Meta-analysis: audit and feedback features impact effectiveness on care quality. Med Care. 2009;47:356-63. https://doi.org/10.1097/MLR. Ob013e3181893f6b.

32. O'Brien MA, Rogers $\mathrm{S}$, Jamtvedt $\mathrm{G}$, Oxman AD, Odgaard-Jensen J, Kristoffersen DT, Forsetlund L, Bainbridge D, Freemantle N, Davis DA, Haynes RB, Harvey EL. Educational outreach visits: effects on professional practice and health care outcomes. Cochrane Database Syst Rev. 2007;(4): CD000409. https://www.ncbi.nlm.nih.gov/pubmed/17943742.

33. Reszel J, Dunn SI, Sprague AE, Graham ID, Grimshaw JM, Peterson WE, et al. Use of a maternal newborn audit and feedback system in Ontario: a collective case study. BMJ Qual Saf. 2019. https://doi.org/10. 1136/bmjgs-2018-008354

34. Gude WT, van der Veer SN, de Keizer NF, Coiera E, Peek N. Optimizing digital health informatics interventions through unobtrusive quantitative process evaluations. Stud Health Technol Inform. 2016;228:594-8.

35. Scott PJ, Rigby M, Ammenwerth E, McNair JB, Georgiou A, Hyppönen $H$, et al. Evaluation considerations for secondary uses of clinical data: principles for an evidence-based approach to policy and implementation of secondary analysis. Yearb Med Inform. 2017;26:59-67. https://doi.org/10, 15265/IY-2017-010.

36. Grant A, Treweek S, Dreischulte T, Foy R, Guthrie B. Process evaluations for cluster-randomised trials of complex interventions: a proposed framework for design and reporting. Trials. 2013;14:15. https://doi.org/10. 1186/1745-6215-14-15.

\section{Publisher's Note}

Springer Nature remains neutral with regard to jurisdictional claims in published maps and institutional affiliations.
Ready to submit your research? Choose BMC and benefit from:

- fast, convenient online submission

- thorough peer review by experienced researchers in your field

- rapid publication on acceptance

- support for research data, including large and complex data types

- gold Open Access which fosters wider collaboration and increased citations

- maximum visibility for your research: over $100 \mathrm{M}$ website views per year

At BMC, research is always in progress.

Learn more biomedcentral.com/submissions 